\title{
Effect of Feeding Low-Oil Ddgs to Laying Hens and Broiler Chickens on Performance and Egg Yolk and Skin Pigmentation
}

\section{-Author(s)}

Cortes-Cuevas A

Ramírez-Estrada S

Arce-Menocal J"

Avila-González E'

López-Coello $\mathrm{C}^{\prime}$

Facultad de Medicina Veterinaria y Zootecnia, Universidad Nacional Autónoma de México.

" Facultad de Medicina Veterinaria y Zootecnia, Universidad Michoacana de San Nicolás de Hidalgo.

\section{Mail Address}

Corresponding author e-mail address Cuevas AC

Manuel M. Lopez s/n, Colonia Zapotitlan 13209, Mexico, México D.F, Mexico

Phone: (55) 58451530

E-mail: cortescuevasarturo@yahoo.com

\section{Eeywords}

Low-oil DDGS, egg yolk pigmentation, skin pigmentation, abdominal fat, carcass yield.

\section{SUMMARY}

Two experiments were conducted to evaluate the nutritional quality of two sources of low-oil distiller's dried grains with solubles (DDGS) and their pigmenting ability for broiler chicken skin and egg yolks. In Experiment 1, 360 Bovan-White hens between 69 and 77 weeks of age were randomly assigned to five dietary treatments with 6 replicates of 12 hens each. In Experiment 2, 375 Ross 308 broiler chickens were randomly assigned to five treatments with three replicates of 25 birds each. The chickens were fed the experimental diets from one to 42 $\mathrm{d}$ of age. In both experiments, treatments consisted of a basal diet with no DDGS, and diets with $6 \%$ or $12 \%$ inclusion of DDGS from two sources. In Experiment 1, no significant differences in performance were detected among treatments ( $p>0.05$ ). Egg yolk pigmentation, according to CR-400 Minolta Colorimeter redness (a) and yellowness (b), linearly increased $(p<0.05)$ with DDGS inclusions. In Experiment 2, no significant differences ( $p>0.05$ ) were detected among treatments in growth performance, carcass yield, or abdominal fat at $42 \mathrm{~d}$ of age. Yellowness linearly increased $(p<0.05)$ in the skin and abdominal fat of the birds that consumed diets with DDGS. The results of the current study indicate that feeding two sources of low-oil DDGS to broiler chicks or laying hens does not negatively affect egg production or growth performance while improves egg yolk and skin yellowness.

\section{INTRODUCTION}

After the fermentation process of corn to produce ethanol, the remaining fat in the by-product distiller's dried grains with solubles (DDGS) is extracted by centrifugation. The resulting by-product is known as low-fat DDGS, which has slightly higher protein content, amongst other nutrients, compared with conventional DDGS (Kshun and Kurt, 2012). The fat extraction in low-oil DDGS reduces its xanthophyll content in comparison with the conventional DDGS (Winkler and Vaughn, 2009). Lower concentrations (10-30 mg/g) of lutein and zeaxanthin have been analyzed in low-oil DDGS with respect to conventional DDGS sources $(25-50 \mathrm{mg} / \mathrm{kg}$ ) (Winkler and Vaughn, 2009; Moreau et al., 2010).

Conventional DDGS can be included up to $15 \%$ in layer diets and increase egg yolk pigmentation. When increasing the DDGS inclusion in diets for second-cycle Bovan-White birds, egg yolk pigmentation was more intense (Loar et al 2010). In broiler chickens fed diets with $0,6,12,18$, or $24 \%$ inclusion of conventional DDGS, there were no significant changes in breast skin pigmentation after cooking (Schilling et al., 2010). However, there are no research reports on the pigmenting ability of low-oil DDGS for egg yolk or broiler skin, which are important traits for commercialization of eggs and chicken meat in the Mexican market. 
The objective of the current study was to evaluate the effect of feeding two low-oil DDGS sources on egg production, broiler growth performance, carcass yield, and egg yolk and skin pigmentation.

\section{MATERIAL AND METHODS}

\section{Laboratory analysis}

Proximate analysis of the DDGS samples was performed by wet chemistry using the AOAC methods (2006) (Table 1). Total xanthophylls, lutein, and zeaxanthin were determined through HPLC using a Hewlett-Packard 1100 chromatographer comprising a quaternary pump, a degasifier, an automatic injector, and variable-wave length detector. The UV absorbance was recorded at $450 \mathrm{~nm}$. A C30 reversed phase analytical column (RP-C30; $250 \times 4.6 \mathrm{~mm}, 5 \mathrm{~m}$ ) and a Bischoff pre-column (Nucleosil C18, $10 \times 4.6 \mathrm{~mm}$, $5 \mathrm{~m})$ temperized at $35^{\circ} \mathrm{C}$ were used. The mobile phase consisted of methanol, TBME (Tert Butyl Metyl Ether), and water; solvent $A$ : 81:15:4( $\mathrm{vol} / \mathrm{vol} / \mathrm{vol})$; solvent B: 6:90:4(vol/vol/vol)]. The following gradient was applied: (minutes over percent of solvent $A$ ): 0/99, 39/44, 45/0, 50/99, 55/99) with a flow of $1 \mathrm{~mL}$ min; injection volumen was 20L. Analytical data were collected using the HP-ChemStation Plus software. The HPLC was conducted in an LC (APCl)-MS system coupled to a Micromass VG platform II quadruple mass spectrophotometer, which was operated with an APcl interphase on a positive mode. The MS parameters were as described by Breithaunpt et al. (2002). Analytical results are presented in Table 1.

Table 1 - Nutrient composition of two low-oil DDGS sources.

\begin{tabular}{lcc}
\hline Nutrient & 1DDGS A & 2DDGS B \\
\hline Dry matter & 95.45 & 95.05 \\
\hline Moisture & 4.55 & 4.95 \\
\hline Crude protein & 28.05 & 27.02 \\
\hline Ether extract & 6.54 & 5.39 \\
\hline Ash & 5.40 & 5.26 \\
\hline Crude fiber & 8.05 & 8.43 \\
\hline Nitrogen-free extract & 47.41 & 48.96 \\
\hline Total xanthophylls $(\mathrm{mg} / \mathrm{kg})$ & 24.2 & 25.6 \\
\hline Lutein (mg/kg) & 5.9 & 6.0 \\
\hline Zeaxanthin (mg/kg) & 8.1 & 10.3 \\
\hline Lysine \% & 1.00 & 0.98 \\
\hline Met+Cyst \% & 1.03 & 1.06 \\
\hline Lysine Dig. \% & 0.65 & 0.64 \\
\hline Met+Cyst. Dig \% & 0.84 & 0.85 \\
\hline
\end{tabular}

\section{Bird husbandry}

For both experiments, housing, husbandry, and euthanasia procedures were approved by the institutional committee for experimental animal care of the Faculty of Veterinary Medicine at the National Autonomous University of Mexico (UNAM). Birds were housed in an open-sided, naturally ventilated house.

Experiment 1. The objective of this experiment was to evaluate the effect of two low-oil DDGS samples included at $6 \%$ and $12 \%$ in sorghum-soybean meal diets for Bovans-White laying hens on egg production and egg yolk pigmentation. Three hundred and sixty 69-week-old Bovans White caged hens were randomly assigned to five dietary treatments with six replicates of 12 birds each (3 birds per cage). The birds received the experimental feed in the mash form, and had free access to feed and water.

Treatments consisted of: 1) basal sorghum-soybean meal diet containing $8 \mathrm{ppm}$ of xanthophylls from Tagetes erecta; 2) As $1+6 \%$ DDGS source $A$; 3) As 1 $+12 \%$ DDGS source $A$; 4) As $1+6 \%$ DDGS source $B$; and 5) As $1+12 \%$ DDGS source $B$. The composition of the experimental diets is presented in Table 2 . The xanthophyll content in diets of treatments 2 through 5 was higher as the inclusion of DDGS increased.

The hens received the experimental diets for 8 weeks. Egg production, egg mass, feed intake, and feed conversion ratio were recorded weekly. In weeks 4 and 8 of the experimental period, egg yolk coloration was visually evaluated using DSM's egg yolk color fan. $\left(L^{*}\right)$ Luminosity, redness $\left(a^{*}\right)$, and yellowness $\left(b^{*}\right)$ were also determined using a Minolta CR-400 colorimeter.

Experiment 2. The objective was to evaluate the effect of feeding two low-oil DDGS sources included at $6 \%$ and $12 \%$ in sorghum-soybean meal diets on the growth performance, carcass yield, skin pigmentation, and abdominal fat of broilers. Three hundred and seventy five 1-d-old Ross 308 male broiler chickens were randomly assigned to five dietary treatments with three replicates of 25 birds each. The birds were housed in an open-sided, naturally-ventilated house with floor pens, bell-type drinkers, feed troughs, and automatic gas brooders.

The composition of the experimental diets is presented in Tables 3, 4, and 5. Diets were designed for three growth phases: starter (0-11 d), grower (11-21 d), and finisher (22-42 d), and formulated to meet the nutrient requirements as recommended by the primary breeder (Ross 308, 2012) [9]. Treatments consisted of the following: 1) basal sorghum-soybean meal diet containing 8 ppm of xanthophylls from Tagetes erecta; 
Table 2 - Composition of the experimental diets for Bovans-White hens from 69 to 77 weeks (Exp. 1)

\begin{tabular}{|c|c|c|c|c|c|}
\hline Ingredient & Control & $\begin{array}{c}6 \% \\
\text { DDGS A }\end{array}$ & $\begin{array}{c}12 \% \\
\text { DDGS A }\end{array}$ & $6 \%$ DDGS B & $12 \%$ DDGS B \\
\hline Sorghum & 697.436 & 665.843 & 634.216 & 664.440 & 630.857 \\
\hline Soybean meal & 178.175 & 150.124 & 120.976 & 149.039 & 119.935 \\
\hline Calcium carbonate & 90.201 & 90.923 & 91.646 & 91.109 & 92.017 \\
\hline Monodicalcium phosphate & 16.063 & 14,659 & 13.268 & 14.513 & 12.963 \\
\hline Soy oil & 8.371 & 9.166 & 10.017 & 11.064 & 13.812 \\
\hline Salt & 3.762 & 3.818 & 3.875 & 3.820 & 3.878 \\
\hline DL-Methionine & 1.475 & 1.384 & 1.308 & 1.396 & 1.318 \\
\hline Vitamins* & 1.000 & 1.000 & 1.000 & 1.000 & 1.000 \\
\hline L-Lysine $\mathrm{HCl}$ & 0.652 & 0.716 & 1.329 & 1.254 & ------- \\
\hline Yellow pigment** & 0.615 & 0.615 & 0.615 & 0.615 & 0.615 \\
\hline Minerals* & 0.500 & 0.500 & 0.500 & 0.500 & 0.500 \\
\hline Bacitracin 10\% & 0.300 & 0.300 & 0.300 & 0.300 & 0.300 \\
\hline Antioxidant & 0.150 & 0.150 & 0.150 & 0.150 & 0.150 \\
\hline Choline chloride $60 \%$ & 0.800 & 0.800 & 0.800 & 0.800 & 0.800 \\
\hline DDGS & -------- & 60.00 & 120.00 & 60.00 & 120.00 \\
\hline Total & 1000.0 & 1000.0 & 1000.0 & 1000.0 & 1000.0 \\
\hline Nutrient & \multicolumn{5}{|c|}{ Calculated analysis } \\
\hline Crude protein (\%) & 15.00 & 15.00 & 15.00 & 15.00 & 15.00 \\
\hline ME (Kcal/Kg) & 2800 & 2800 & 2800 & 2800 & 2800 \\
\hline Calcium (\%) & 4.00 & 4.00 & 4.00 & 4.00 & 4.00 \\
\hline Available phosphorus (\%) & 0.400 & 0.400 & 0.400 & 0.400 & 0.400 \\
\hline Digestible lysine (\%) & 0.670 & 0.670 & 0.670 & 0.670 & 0.670 \\
\hline Dig Met-Cys (\%) & 0.580 & 0.580 & 0.580 & 0.580 & 0.580 \\
\hline Sodium (\%) & 0.180 & 0.180 & 0.180 & 0.180 & 0.180 \\
\hline Xanthophylls (mg/Kg) & 8.000 & 8.000 & 8.000 & 8.000 & 8.000 \\
\hline
\end{tabular}

*Provides per kg: Vitamin A, 3000000 Ul; Vitamin D3, 750000 Ul; Vitamin E, 6000 Ul; Vitamin K3, 1.0 g; Riboflavin, 4 g; B12, 0.060 g; Pyridoxin, 3.0 g; Calcium pantothenate, 13.0 g; Niacin, 25 g; Biotin, 0.063 g; Choline chloride, 250 g.; selenium, 0.2 g; cobalt, 0.1 g; iodine, 0.3 g; copper, 10 g; zinc, 50 g; iron, 100 g; manganese, 100 g; carrier to 1000 g.

** 8 ppm of yellow pigment added.

2) As $1+6 \%$ DDGS source $A$; 3) As $1+12 \%$ DDGS source $A$; 4) As $1+6 \%$ DDGS source $B$; and 5) As $1+$ $12 \%$ DDGS source $B$.

The birds received the experimental diets for 42 $d$, and live weight, feed intake, and feed conversion ratio were recorded weekly. At the end of the study, 12 birds per treatment (six males and six females) were euthanized by $\mathrm{CO}_{2}$ asphyxiation. The carcasses were eviscerated, defeathered, and head, neck, and feet removed. Carcass weight was used to calculate carcass yield. Abdominal fat was collected and weighed. Yellowness $\left(b^{*}\right)$ was measured on the lateral apterial breast area using the CR-400 Minolta colorimeter in live birds, hot and cold carcasses, and abdominal fat.

\section{Statistical analysis}

All collected data were submitted to analysis of variance (ANOVA) using the SPSS software for Windows, version 17.0 (2012). If a significant difference was detected $(p<0.05)$, treatment means were compared using Tukey's multiple comparison procedure. Skin pigmentation in live birds, hot and cold carcass, and abdominal fat weight and carcass yield were analyzed using a $3 \times 2$ factorial arrangement, where one factor was the inclusion of DDGS $(0 \%, 6 \%$, and $12 \%)$ and the other factor was the bird's sex (male or female).

\section{RESULTS AND DISCUSSION}

Coloration data for the two DDGS samples is presented in Table 2. Luminosity as measured by the Minolta CR-400 colorimeter $\left(L^{*}\right)$ was significantly higher in the DDGS source with the lower ether extract value $(p<0.05)$. However, the redness $\left(a^{*}\right)$ and yellowness $\left(b^{*}\right)$ values were significantly greater in the DDGS source with higher ether extract $(p<0.05)$. 
Table 3 - Composition of the starter diets (0-10 d) for broiler chickens, Experiment 2.

\begin{tabular}{|c|c|c|c|c|c|}
\hline Ingredient & $\begin{array}{c}\text { Diet } \\
\text { control }\end{array}$ & $6 \%$ DDGS A & $12 \%$ DDGS A & $6 \%$ DDGS B & $12 \%$ DDGS B \\
\hline Sorghum & 557.34 & 526.29 & 495.25 & 523.84 & 490.35 \\
\hline Soybean meal & 365.76 & 336.39 & 307.03 & 336.62 & 307.49 \\
\hline DDGS & 0.00 & 60.00 & 120.00 & 60.00 & 120.00 \\
\hline Soy oil & 31.29 & 31.73 & 32.24 & 33.97 & 36.70 \\
\hline Monodicalcium phosphate & 20.10 & 18.71 & 17.31 & 18.55 & 17.00 \\
\hline Calcium carbonate & 13.45 & 14.17 & 14.89 & 14.35 & 15.26 \\
\hline Salt & 3.790 & 3.840 & 3.900 & 3.840 & 3.900 \\
\hline L-Lysine $\mathrm{HCl}$ & 2.090 & 2.700 & 3.320 & 2.690 & 3.290 \\
\hline DL-Methionine 99\% & 3.240 & 3.160 & 3.090 & 3.160 & 3.090 \\
\hline Choline chloride $60 \%$ & 1.000 & 1.000 & 1.000 & 1.000 & 1.000 \\
\hline Vitamins* & 1.000 & 1.000 & 1.000 & 1.000 & 1.000 \\
\hline Minerals* & 0.500 & 0.500 & 0.500 & 0.500 & 0.500 \\
\hline Bacitracin 10\% & 0.300 & 0.300 & 0.300 & 0.300 & 0.300 \\
\hline Antioxidant & 0.150 & 0.150 & 0.150 & 0.150 & 0.150 \\
\hline Total & 1000 & 1000 & 1000 & 1000 & 1000 \\
\hline Nutrient & \multicolumn{4}{|c|}{ Calculated analysis } & \\
\hline $\mathrm{ME}(\mathrm{Kcal} / \mathrm{Kg})$ & 3025 & 3025 & 3025 & 3025 & 3025 \\
\hline Crude protein (\%) & 23.00 & 23.00 & 23.00 & 23.00 & 23.00 \\
\hline Dig Met+ Cyst (\%) & 0.94 & 0.94 & 0.94 & 0.94 & 0.94 \\
\hline Digestible lysine (\%) & 1.27 & 1.27 & 1.27 & 1.27 & 1.27 \\
\hline Calcium & 1.05 & 1.05 & 1.05 & 1.05 & 1.05 \\
\hline Available phosphorus & 0.50 & 0.50 & 0.50 & 0.50 & 0.50 \\
\hline
\end{tabular}

Similar results have been reported elsewhere (Pekel et al., 2013), where higher fat content was associated with higher yellowness.

Experiment 1. The inclusion of DDGS at $6 \%$ and $12 \%$ did not affect egg production, egg weight, egg mass, feed intake, or feed conversion ratio of Bovans-White hens (Table 6). Research conducted elsewhere (Roberson et al., 2005; Cheon et al., 2008; Lumpkins et al., 2005; Wu-Haan et al., 2010) indicated no detrimental effects on hen performance when conventional DDGS with $10 \%$ fat were included between $5 \%$ and $20 \%$ of the diet. In the study reported herein, DDGS inclusion was within the range of inclusion reported in the published literature. Inclusion levels of DDGS as high as $32 \%$ had no detrimental effects on hen performance (Loar et al., 2010).

Feeding a low-oil DDGS source (5.62\% fat) did not reduce hen performance when included in diets at levels as high as 20\% (Noll and Purdum, 2013), which is in agreement with the results observed in the study reported herein.
Egg yolk coloration data are presented in Table 7. Luminosity was not significantly influenced by DDGS inclusion level or source ( $p>0.05)$. In a previous study, higher DDGS inclusion was reflected in lower egg yolk luminosity (Loar et al., 2010). Redness (a*) significantly increased with higher inclusion of DDGS from both sources, which is in agreement with previous research, where a positive linear relationship between egg yolk redness and DDGS inclusion was found (Robertson et al., 2005).

Egg yolk color score and yellowness $\left(b^{*}\right)$ linearly increased with increasing DDGS inclusion from both sources $(p<0.001)$. This effect is attributed to the greater xanthophyll contribution (lutein and zeaxanthin) from DDGS with respect to the control diet. This effect has been documented in previous research studies (Cheon et al., 2008; Masa'deh et al., 2011; Cortés et al., 2012; Sun et al., 2013) using conventional DDGS sources. The results of the current study indicate that fat extraction in DDGS did not affect the egg yolk pigmenting capacity of this ingredient. 
Table 4 - Composition of the grower diets (11-21 d) for broiler chickens, Experiment 2

\begin{tabular}{|c|c|c|c|c|c|}
\hline Ingredient & Control & $6 \%$ DDGS A & $12 \%$ DDGS A & $\begin{array}{c}6 \% \text { DDGS } \\
\text { B }\end{array}$ & $12 \%$ DDGS B \\
\hline Sorghum & 597.49 & 566.45 & 535.41 & 593.90 & 530.50 \\
\hline Soybean meal & 317.97 & 288.60 & 259.24 & 288.83 & 259.69 \\
\hline DDGS & 0.00 & 60.00 & 120.00 & 60.00 & 120.00 \\
\hline Soy oil & 44.74 & 42.23 & 45.70 & 47.44 & 50.13 \\
\hline Monodicalcium phosphate & 17.69 & 16.30 & 14.91 & 16.15 & 14.59 \\
\hline Calcium carbonate & 11.14 & 11.87 & 12.59 & 12.05 & 12.96 \\
\hline Salt & 3.810 & 3.870 & 3.930 & 3.870 & 3.930 \\
\hline L-Lysine $\mathrm{HCl}$ & 1.490 & 2.110 & 2.730 & 2.090 & 2.690 \\
\hline DL-Methionine 99\% & 2.690 & 2.620 & 2.540 & 2.620 & 2.540 \\
\hline Choline chloride $60 \%$ & 1.000 & 1.000 & 1.000 & 1.000 & 1.000 \\
\hline Vitamins* & 1.000 & 1.000 & 1.000 & 1.000 & 1.000 \\
\hline Minerals* & 0.500 & 0.500 & 0.500 & 0.500 & 0.500 \\
\hline Bacitracin $10 \%$ & 0.300 & 0.300 & 0.300 & 0.300 & 0.300 \\
\hline Antioxidant & 0.150 & 0.150 & 0.150 & 0.150 & 0.150 \\
\hline Total & 1000 & 1000 & 1000 & 1000 & 1000 \\
\hline Nutrient & \multicolumn{4}{|c|}{ Calculated analysis } & \\
\hline ME (Kcal/Kg) & 3150 & 3150 & 3150 & 3150 & 3150 \\
\hline Crude protein (\%) & 21.00 & 21.00 & 21.00 & 21.00 & 21.00 \\
\hline Dig Met+ Cyst (\%) & 0.84 & 0.84 & 0.84 & 0.84 & 0.84 \\
\hline Digestible lysine (\%) & 1.10 & 1.10 & 1.10 & 1.10 & 1.10 \\
\hline Calcium & 0.90 & 0.90 & 0.90 & 0.90 & 0.90 \\
\hline Available phosphorus & 0.45 & 0.45 & 0.45 & 0.45 & 0.45 \\
\hline
\end{tabular}

By increasing the inclusion of DDGS in the experimental diets, it was possible to augment the total xanthophyll contribution by 1.40-1.54 ppm xanthophylls from source $A$, and 2.90-3.08 ppm from source $B$, respectively. The control diet was formulated to contain 8 ppm of total xanthophylls.

Experiment 2. Broiler growth performance data are presented in Table 8. The inclusion of low-fat DDGS at $0 \%, 6 \%$, or $12 \%$ had no detrimental effect on growth performance. There was no significant difference between DDGS sources either ( $p>0.05)$. Similare results have been reported elsewhere (Guney et al., 2013) when broilers were fed low-oil DDGS for $18 \mathrm{~d}$. Feeding levels as high as $15 \%$ of conventional DDGS $(10 \%$ fat) did not significantly affect growth performance (Cortés et al., 2012; Lumpkins et al., 2004; Shim et al., 2011). Levels higher than $15 \%$, however, significantly reduced growth performance rate. This reduction in growth performance was attributed to an amino acid imbalance created by reducing soybean meal inclusion, which has a better amino acid profile than DDGS.
Carcass yield results and abdominal fat weight data are presented in Table 9. No significant effect was detected for either DDGS inclusion or source. Similar results were reported by Lu and Chen (2005), who did not find any significant difference in the carcass yield of broilers fed corn-soybean meal diets with the inclusion of $10 \%$ or 20\% conventional DDGS. Loar et al. (2012), however, reported reduced carcass yield when DDGS inclusion was higher than $14 \%$ only in the finisher phase.

The dietary inclusion of low-oil DDGS did not affect abdominal fat weight. Guney et al. (2013), on the other hand, reported a reduction in abdominal fat weight when low-fat DDGS was fed at $10 \%$ or $20 \%$ inclusion level.

There was no significant effect of sex on carcass yield or abdominal fat weight ( $p>0.05$ ). In contrast, Shim et al. (2011) reported that female broilers accumulated more abdominal fat than males.

Data for skin pigmentation of live birds and pre-chill carcasses, as well as abdominal fat pigmentation, are presented in Table 10. The consumption of 272.4 and 
Table 5 - Composition of the finisher diets (22-42 d) for broiler chickens.

\begin{tabular}{|c|c|c|c|c|c|}
\hline Ingredient & Control & $6 \%$ DDGS A & $\begin{array}{c}12 \% \text { DDGS } \\
\mathrm{A}\end{array}$ & $6 \%$ DDGS B & $12 \%$ DDGS B \\
\hline Sorghum & 639.2 & 608.161 & 577.119 & 605.700 & 572.200 \\
\hline Soybean meal & 270.0 & 240.689 & 211.326 & 240.900 & 211.800 \\
\hline DDGS & 0.000 & 60.000 & 120.000 & 60.000 & 120.000 \\
\hline Soy oil & 49.20 & 49.695 & 50.175 & 51.900 & 54.600 \\
\hline Monodicalcium phosphate & 16.40 & 15.006 & 13.612 & 14.800 & 13.300 \\
\hline Calcium carbonate & 10.70 & 11.515 & 12.239 & 11.700 & 12.600 \\
\hline Yellow pigment* & 5.000 & 5.000 & 5.000 & 5.000 & 5.000 \\
\hline Salt & 5.000 & 3.372 & 3.429 & 5.000 & 5.000 \\
\hline L-Lysine $\mathrm{HCl}$ & 3.300 & 1.644 & 2.262 & 3.400 & 3.400 \\
\hline DL-Methionine 99\% & 1.000 & 2.432 & 2.336 & 1.600 & 2.200 \\
\hline Choline chloride $60 \%$ & 2.500 & 1.000 & 1.000 & 2.400 & 2.300 \\
\hline Vitamins** & 1.000 & 1.000 & 1.000 & 1.000 & 1.000 \\
\hline Minerals** & 1.000 & 0.500 & 0.500 & 1.000 & 1.000 \\
\hline Bacitracin $10 \%$ & 0.500 & 0.300 & 0.300 & 0.500 & 0.500 \\
\hline Antioxidant & 0.300 & 0.150 & 0.150 & 0.300 & 0.300 \\
\hline Total & 1000 & 1000 & 1000 & 1000 & 1000 \\
\hline \multicolumn{6}{|l|}{ Calculated analysis } \\
\hline $\mathrm{ME}(\mathrm{Kcal} / \mathrm{Kg})$ & 3200 & 3200 & 3200 & 3200 & 3200 \\
\hline Crude protein (\%) & 19 & 19 & 19 & 19 & 19 \\
\hline Dig Met+ Cyst (\%) & 0.73 & 0.73 & 0.73 & 0.73 & 0.73 \\
\hline Digestible lysine (\%) & 0.94 & 0.94 & 0.94 & 0.94 & 0.94 \\
\hline Calcium & 0.85 & 0.85 & 0.85 & 0.85 & 0.85 \\
\hline Available phosphorus & 0.42 & 0.42 & 0.42 & 0.42 & 0.42 \\
\hline
\end{tabular}

* 70 ppm of yellow pigment (Tagetes erecta).

**Provided per kg. Vitamin A, 6000000 Ul; Vitamin D3, 1,500 000 Ul; Vitamin E, 12000 Ul; Vitamin K3, 2.0 g; Riboflavin, 8 g; B12, 0.120 g; Pyridoxin, 6.0 g; Calcium pantothenate, 26.0 g; Niacin, 50 g; Biotin, 0.126 g; Choline chloride, 500 g; selenium, 0.2 g; cobalt, 0.1 g; iodine, 0.3 g; copper, 10 g; zinc, 50 g; iron, 100 g; manganese, $100 \mathrm{~g}$; carrier to $2000 \mathrm{~g}$.

278.7 ppm of xanthophylls from $6 \%$ or $12 \%$ DDGS from source B produced greater skin pigmentation than the consumption of DDGS from source A (260.3 and 269.8 $\mathrm{ppm}$, respectively). Birds from the control group had a calculated xanthophyll consumption of $260.2 \mathrm{ppm}$. No significant difference was detected in skin pigmentation between the two levels of DDGS inclusion.

Skin pigmentation in hot carcasses was not different ( $p>0.05)$ between the two DDGS sources. However, the dietary inclusion of DDGS resulted in greater skin pigmentation than that observed in birds in the control group, regardless of the DDGS source. Similar results were documented by Min et al. (2012), where DDGS inclusion levels as high as 20\% significantly increased skin pigmentation. However, those authors did not observe any effect of $6 \%$ DDGS inclusion. In contrast with these results, the inclusion of DDGS at $6,8,12$, 18 , or $24 \%$ did not have any significant effect on skin

Table 6 - Egg production (\%), egg weight (g/egg), feed consumption (g/bird/d), feed conversion (kg:kg), and egg mass (g/ bird) of hens fed 0\%,6\%, and 12\% inclusion of low-oil DDGS from 2 sources from 69 to 77 weeks of age (Experiment 1).

\begin{tabular}{lccccc}
\hline Treatment & $\begin{array}{c}\text { Egg production } \\
(\%)\end{array}$ & Egg weight $(\mathrm{g})$ & Feed intake $(\mathrm{g})$ & $\begin{array}{c}\text { Feed conversion } \\
\text { ratio }(\mathrm{kg}: \mathrm{kg})\end{array}$ & $\begin{array}{c}\text { Egg mass }(\mathrm{g}) \\
\text { 0\% DDGS }\end{array}$ \\
\hline $6 \% \pm 1.25$ & $64.6 \pm 0.54$ & $108 \pm 1.07$ & $1.93 \pm 0.02$ & $56 \pm 1.03$ \\
\hline $12 \%$ DDGS A & $87 \pm 1.83$ & $64.7 \pm 0.23$ & $109 \pm 0.77$ & $1.94 \pm 0.03$ & $56 \pm 1.24$ \\
\hline $6 \%$ DDGS B & $88 \pm 1.03$ & $64.4 \pm 0.60$ & $107 \pm 1.05$ & $1.91 \pm 0.02$ & $56 \pm 0.36$ \\
\hline $12 \%$ DDGS B & $88 \pm 1.09$ & $65.0 \pm 0.31$ & $109 \pm 0.70$ & $1.92 \pm 0.02$ & $57 \pm 0.66$ \\
\hline Probability & $86 \pm 0.89$ & $64.6 \pm 0.28$ & $108 \pm 1.06$ & $0.96 \pm 0.03$ & $56 \pm 0.71$ \\
\hline
\end{tabular}


Table 7 - Luminosity $\left(L^{*}\right)$, redness $\left(a^{*}\right)$, yellowness $\left(b^{*}\right)$, and color score (DSM egg yolk color fan) in egg yolks of hens fed $0 \%, 6 \%$, and $12 \%$ inclusion of low-oil DDGS from 2 sources from 69 to 77 weeks of age (Experiment 1)

\begin{tabular}{lllllll}
\hline Parameter & $0 \%$ DDGS & $6 \%$ DDGS A & $12 \%$ DDGS A & $6 \%$ DDGS B & $12 \%$ DDGS B & Probability \\
\hline $\mathrm{L}^{*}$ & $57.83^{\mathrm{a}} \pm 5.4$ & $65.01^{\mathrm{a}} \pm 0.9$ & $64.63^{\mathrm{a}} \pm 0.9$ & $61.21 \pm 1.9^{\mathrm{a}}$ & $64.66^{\mathrm{a}} \pm 0.7$ & 0.260 \\
\hline $\mathrm{a}^{*}$ & $-7.30^{\mathrm{b}} \pm 0.09$ & $-6.76^{\mathrm{ab}} \pm 0.2$ & $-6.11^{\mathrm{a}} \pm 0.14$ & $-6.69 \pm 0.3^{\mathrm{ab}}$ & $-6.17^{\mathrm{a}} \pm 0.1$ & 0.001 \\
\hline $\mathrm{b}^{*}$ & $36.47^{\mathrm{b}} \pm 2.57$ & $40.15^{\mathrm{ab}} \pm 1.0$ & $42.68 \pm 1.0^{\mathrm{a}}$ & $39.60 \pm 0.7^{\mathrm{ab}}$ & $42.93^{\mathrm{a}} \pm 1.1$ & 0.030 \\
\hline Color score & $3.33^{\mathrm{a}} \pm 0.21$ & $4.08^{\mathrm{ab}} \pm 0.1$ & $4.16^{\mathrm{b}} \pm 0.16$ & $4.00^{\mathrm{ab}} \pm 0.25$ & $4.66^{\mathrm{b}} \pm 0.21$ & 0.002 \\
\hline
\end{tabular}

Means with different superscript within columns (a-c) differ significantly $(p<0.05)$.

Table 8 - Growth performance of broiler chickens fed diets with 0\%,6\%, and 12\% inclusion of two sources of low-oil DDGS from 0 to $42 \mathrm{~d}$ of age (Experiment 2)

\begin{tabular}{lcccc}
\hline Treatment & Final weight $(\mathrm{g})$ & Weight gain $(\mathrm{g})$ & Feed intake $(\mathrm{g})$ & Feed conversion ratio $(\mathrm{kg}: \mathrm{kg})$ \\
\hline $0 \%$ DDGS & $2758 \pm 32$ & $2717 \pm 32$ & $4847 \pm 36$ & $1.78 \pm 0.03$ \\
\hline $6 \%$ DDGS A & $2692 \pm 32$ & $2651 \pm 49$ & $4826 \pm 63$ & $1.82 \pm 0.04$ \\
\hline $12 \%$ DDGS A & $2627 \pm 49$ & $2585 \pm 80$ & $4725 \pm 130$ & $1.83 \pm 0.09$ \\
\hline $6 \%$ DDGS B & $2699 \pm 61$ & $2658 \pm 61$ & $4861 \pm 154$ & $1.83 \pm 0.05$ \\
\hline $12 \%$ DDGS B & $2636 \pm 72$ & $2595 \pm 72$ & $4929 \pm 75$ & $1.90 \pm 0.06$ \\
\hline Probability & 0.495 & 0.490 & 0.691 & 0.709 \\
\hline
\end{tabular}

pigmentation, according to other research reports (Schilling et al., 2010; Corzo et al., 2009).

Table 9 - Carcass yield and abdominal fat weight of broiler chickens at $42 \mathrm{~d}$ of age after receiving diets with $0 \%, 6 \%$, and $12 \%$ inclusion of two sources of low-oil DDGS from 0 to $42 \mathrm{~d}$ of age (Experiment 2).

\begin{tabular}{|c|c|c|c|}
\hline & Male & Female & Average \\
\hline Treatment & \multicolumn{3}{|c|}{ Carcass yield (\%) } \\
\hline $0 \%$ DDGS & 71.5 & 73.5 & $72.5 \pm 0.7$ \\
\hline $6 \%$ DDGS A & 70.6 & 71.0 & $70.8 \pm 0.3$ \\
\hline $12 \%$ DDGS A & 70.5 & 71.8 & $71.1 \pm 0.6$ \\
\hline $6 \%$ DDGS B & 71.7 & 70.7 & $71.2 \pm 0.5$ \\
\hline $12 \%$ DDGS B & 70.9 & 71.8 & $71.3 \pm 0.2$ \\
\hline \multirow[t]{2}{*}{ Average } & $71.1 \pm 0.3$ & $71.8 \pm 0.3$ & \\
\hline & & \multicolumn{2}{|c|}{ Abdominal fat weight (g) } \\
\hline $0 \%$ DDGS & 56.7 & 46.7 & $51.7 \pm 3.3$ \\
\hline $6 \%$ DDGS A & 41.0 & 51.3 & $46.2 \pm 2.9$ \\
\hline $12 \%$ DDGS A & 55.3 & 59.7 & $57.5 \pm 1.9$ \\
\hline $6 \%$ DDGS B & 54.3 & 61.2 & $57.7 \pm 4.0$ \\
\hline $12 \%$ DDGS A & 49.7 & 56.0 & $52.8 \pm 4.3$ \\
\hline Average & $51.4 \pm 2.3$ & $55.0=$ & \\
\hline
\end{tabular}

Means with different superscript within rows (a-c) or columns (2-y) differ significantly $(p<0.05)$.

Abdominal fat pigmentation was greater in the birds that consumed diets with 12\% DDGS from both evaluated sources. Abdominal fat pigmentation, however, was significantly greater for source B. Increasing levels of DDGS inclusion have been reported to increase yellowness values $\left(b^{*}\right)$ in abdominal fat (Kimura, 2007).
Table 10 - Skin yellowness $\left(b^{*}\right)$ in broiler chickens at 42 d of age after receiving diets with $0 \%, 6 \%$, and $12 \%$ inclusion of two sources of low-oil DDGS from 0 to $42 \mathrm{~d}$ of age (Experiment 2)

\begin{tabular}{|c|c|c|c|}
\hline & Male & Female & Average \\
\hline Treatment & \multicolumn{3}{|c|}{ Live bird } \\
\hline $0 \%$ DDGS & 11.0 & 13.2 & $12.0^{c} \pm 0.3$ \\
\hline $6 \%$ DDGS A & 12.1 & 13.0 & $12.6^{b c} \pm 0.5$ \\
\hline $12 \%$ DDGSA & 12.5 & 15.1 & $13.9^{\mathrm{ab}} \pm 0.5$ \\
\hline $6 \%$ DDGS B & 13.5 & 14.6 & $14.1^{\mathrm{ab}} \pm 0.4$ \\
\hline $12 \%$ DDGS B & 14.3 & 15.1 & $14.7^{\mathrm{a}} \pm 0.5$ \\
\hline \multirow[t]{2}{*}{ Average } & $12.5 \pm 0.3^{z}$ & $14.2 \pm 0.3^{y}$ & \\
\hline & \multicolumn{3}{|c|}{ Pre-chill carcass } \\
\hline $0 \%$ DDGS & 27.8 & 27.5 & $27.7^{b} \pm 0.8$ \\
\hline $6 \%$ DDGS A & 28.8 & 27.6 & $28.2^{\mathrm{ab}} \pm 0.8$ \\
\hline $12 \%$ DDGS A & 30.1 & 30.2 & $30.2^{\mathrm{ab}} \pm 1.1$ \\
\hline $6 \%$ DDGS B & 31.2 & 30.0 & $30.6^{\mathrm{ab}} \pm 0.7$ \\
\hline $12 \%$ DDGS B & 32.1 & 30.9 & $32.0^{\mathrm{a}} \pm 1.2$ \\
\hline \multirow[t]{2}{*}{ Average } & $30.2 \pm 0.8$ & $29.2 \pm 0.6$ & \\
\hline & \multicolumn{3}{|c|}{ Chilled carcass } \\
\hline $0 \%$ DDGS & 36.4 & 35.5 & $35.9^{\mathrm{a}} \pm 0.7$ \\
\hline $6 \%$ DDGS A & 35.7 & 37.5 & $36.6^{a} \pm 1.2$ \\
\hline $12 \%$ DDGS A & 40.0 & 38.5 & $39.3^{a} \pm 1.0$ \\
\hline $6 \%$ DDGS B & 40.9 & 37.5 & $39.2^{a} \pm 1.4$ \\
\hline $12 \%$ DDGS B & 39.3 & 39.9 & $39.6^{a} \pm 0.8$ \\
\hline \multirow[t]{2}{*}{ Average } & $38.5 \pm 0.6$ & $37.8 \pm 0.8$ & \\
\hline & \multicolumn{3}{|c|}{ Abdominal fat } \\
\hline $0 \%$ DDGS & 20.2 & 21.6 & $20.9^{c} \pm 0.6$ \\
\hline $6 \%$ DDGS A & 21.3 & 21.6 & $21.5^{\mathrm{bc}} \pm 0.8$ \\
\hline $12 \%$ DDGS A & 24.5 & 24.4 & $24.5^{\mathrm{ab}} \pm 0.7$ \\
\hline $6 \%$ DDGS B & 22.6 & 25.0 & $23.8^{\mathrm{abc}} \pm 0.9$ \\
\hline $12 \%$ DDGS A & 27.4 & 23.9 & $25.7^{\mathrm{a}} \pm 1.0$ \\
\hline Average & $23.2 \pm 0.7$ & $23.3 \pm 0.5$ & \\
\hline
\end{tabular}


Skin pigmentation in live birds was significantly higher in females $(p<0.05)$, which may be explained by their higher subcutaneous fat deposition. Nevertheless, there was no effect of sex on pre-chill carcass or abdominal fat pigmentation $(p<0.05)$.

The results of the study reported herein indicated that production performance in Bovans White laying hens and Ross 308 broilers was not affected when fed $6 \%$ or $12 \%$ inclusion of two sources of low-oil DDGS in sorghum-soybean meal-based diets. Egg yolk yellowness linearly increased with two sources. Carcass yield and abdominal fat content were not significantly affected in broilers. Moreover, feeding $6 \%$ or $12 \%$ inclusion of low-oil DDGS from two sources linearly increased skin yellowness in live birds, pre-chill and chilled carcass, and abdominal fat. The findings regarding the improvement on egg yolk and broiler skin pigmentation should be an aid for poultry nutritionists to consider the xanthophyll content of low-oil DDGS, when formulating diets for these poultry species.

\section{REFERENCES}

AOAC International. Official methods of analysis of AOAC International. 18th ed. Gaithersburg: AOAC Internationa; 2006

Aviagen. Ross 308 Broiler: performance objectives. Huntsville; 2012

Breithaupt DE, Wirt U, Bamedi A. Differentiation between lutein monoester regioisomers and detection of lutein diester from marigold flowers (Tagetes erecta L.) and several fruits by liquid chromatography-mass spectrometry. Journal Agriculture Food Chemistry 2002;50(1):66-70.

Cheon $Y$ J, Lee $H L$, Shin $M H$, Jang $A$, Lee SK, Lee JH, Lee BD, Son CK. Effects of Corn Distiller's Dried Grains with Solubles on Production and Egg Quality in Laying Hens. Asian-Australian Journal Animal Science 2008;21(9):1318-1323.

Cortés CA, Esparza CC, Sanabria EG, Miguel JJ, Ornelas RM, Avila GE. El uso de granos secos de destilería con solubles (DDGS) en dietas sorgosoya para pollos de engorda y gallinas de postura. Revista Mexicana de Ciencias Pecuarias 2012;3(3): 331-341.

Corzo A, Schilling MW, Loar RE, Jackson V, Kin S, Radhakrishnan V. The effects of feeding distillers dried grains with solubles on broiler meat quality. Poultry Science 2009;88(11):432-439

Guney AC, Shim MY, Batal AB, Dale NM, Pesti GM. Effect of feeding lowoil distillers dried grains with solubles on the performance of broilers. Poultry Science 2013;92 (8):2070-2076.

Kimura N. Report of the results of a DDGS feeding trial in broilers. Washington: U.S. Grains Council. Laboratory of Animal Nutrition, Nippon Veterinary and Life Science University; 2007

Kshun L, Kurt A. Distillers grains, production, properties, and utilization. AK Boca Raton: CRC Press; 2012.

Loar RE, Donaldson JR, Corzo A. Effects of feeding distillers dried grains with solubles to broilers from 0 to 42 days posthatch on broiler performance, carcass characteristics, and selected intestinal characteristics. Journal Applied Poultry Research 2012;21(1):48-62.
Loar RE, Schilling MW, Daniel MC, Rogers SF, Karges K, Corzo A. Effect of dietary inclusion level of distillers dried grains with solubles on layer performance, egg characteristics, and consumer acceptability. Journal Applied Poultry Research 2010;19 (1):30-37.

Lu JL, Chen Y. Effects of feeding diets containing U.S. corn distiller's dried grains with solubles on growth performance and carcass quality of domestic colored broiler chickens in Taiwan. Taiwan: Dept. of Animal Science, National Chia-Yi University, and AGAPE Nutrition Consultant; 2005.

Lumpkins B, Batal A, Dale N. Use of Distillers Dried Grains Plus Solubles in Laying Hen Diets. Journal Applied Poultry Research 2005;14(1):25-31.

Lumpkins BS, Batal AB, Dale NM. Evaluation of distillers dried grains with solubles as a feed ingredient for broilers. Poultry Science 2004;83(11):1891-1896.

Masa'deh M K, Purdum SE, Hanford KJ. Dried distillers grains with solubles in laying hen diets. Poultry Science 2011;90(9):1960-1966.

Min YN, Li L, Waldroup PW, Niu ZY, Wang ZP, Gao YP, Liu FZ. Effects of dietary distillers dried grains with solubles concentrations on meat quality and antioxidant status and capacity of broiler chickens. Journal Applied Poultry Research 2012;21(3):603-611.

Moreau R, Hicks BK, Johnston BD, Laun NP. The composition of crude corn oil recovered after fermentation via centrifugation from a commercial dry grind ethanol process. Journal American Oil Chemical Society 2010;87(8):895-902

Noll S, Purdum SE. Low oil DDGS value in Turkey and Laying Hen Rations. Minnesota: Midwest Poultry Federation Convention Saint Paul River Centre; 2013.

Pekel AY, Cakır EO, Polat M, Cakır K, İnan G, Kocabaglı N. Correlations between chemical assays and near-infrared reflectance spectroscopy for nutrient components and correlations between nutrients and color scores of distillers dried grains with soluble. Journal Applied Poultry Research 2013;22(4):814-824.

Roberson KD, Kalbfleisch JL, Pan W, Charbeneau RA. Effect of corn distillers dried grains with solubles at various levels on performance of laying hens and egg yolk color. International Journal Poultry Science 2005;4(2):44-51.

Schilling MW, Battula V, Loar RE, Jackson V, Kin S, Corzo A. Dietary inclusion level effects of distillers dried grains with solubles on broiler meat quality. Poultry Science 2010;89(4):752-76.

Shim MY, Pesti GM, Bakalli RI, Tillman PB, Payne RL. Evaluation of corn distillers dried grains with solubles as an alternative ingredient for broiler. Poultry Science 2011;90 (2):369-376.

SPSS. SPSS for Windows (computer program) version 17.0. Chicago: SPSS Inc.; 2012

Sun $H$, Lee EJ, Samaraweera $H$, Persia M, Dong UA. Effects of increasing concentration of corn distillers dried grain with soluble on chemical composition and nutrient content of egg. Poultry Science 2013;92(1):233-242.

Winkler MJ, Vaughn S.F. Antioxidant Activity of Phytochemicals from Distillers Dried Grain Oil. Journal American Oil Chemical Society 2009;86(11):1073-1082

Wu-Haan W, Powers W, Angel R, Applegate TJ. The use of distillers dried grains plus solubles as a feed ingredient on air emissions and performance from laying hens. Poultry Science 2010;89(7):1355-1359. 